\title{
Two dynamic versions of information integration theory for personality impression ${ }^{1}$
}

\author{
YASUHARU OKAMOTO \\ Department of Behavioral Science, Faculty of Letters, Kanazawa University, Marunouchi, Kanazawa 920
}

\begin{abstract}
Dynamic versions of information integration theory by Anderson, N. H. are proposed and applied to two typical type of data, which represent primacy or recency effects. The parameter values were estimated with the method of least squares. Graphical comparison between the data and the predictions indicates that the fitness of the models are very well, however several other factors should be included in future versions of this dynamic type of models.
\end{abstract}

Key words: information integration theory, serial integration, the method of least squares, primacy effect, recency effect.

As a model for serial integration, Anderson (1981, p. 145) proposed the following averaging model,

$$
R=w_{0} \cdot s_{0}+w_{1} \cdot s_{1}+\ldots+w_{N} \cdot s_{N},
$$

where $\sum w_{i}=1$.

Anderson (1965) interpreted that this type of model considers only the final response. However, it seems natural to conceptualize that the subject modifies his previous impression and reconstructs the current one at every step where an additional adjective is presented, especially when the number of the adjectives are large enough to overload his memory capacity. Although Anderson himself also proposed this step-by-step type of model (e.g. Anderson [1959, 1965]), theoretical analysis in his 1981 book is based on the averaging model, not on a step-by-step one. In this article, to examine the plausibility of the step-by-step model, the author specified these types of models to apply to two typical data, one of which represents the primacy effect (Fig. 2.9 in Anderson [1981]) and the other the recency effect (Fig. 3.5 in Anderson [1981]). Most important difference in these experiments is the mode of responding, whether successive or final. That is,

1 Calculations were done at Kanazawa University Data Processing Center. in the final mode the primacy effect was obtained, and in the successive one the recency resulted. Considering effects of the modes of responding and cumulating experience during successive presentations of adjectives, two separate models will be proposed.

In the final mode of responding, the subject responded after all the adjectives were presented. Before the $k$ th presentation, the subject has been exposed to $(k-1)$ adjectives. As $k$ increases, the information of the person builds up. We may assume that the weight of the presented adjective decreases as the number of the adjectives which have been already presented increases. That is, equation (1) is proposed as the model for the final mode of responding in serial integration process,

$$
R_{k}=\frac{w_{0, k} \cdot R_{k-1}+w_{1, k} \cdot S_{k}}{w_{0, k}+w_{1, k}}
$$

where $R_{k}$ is the impression which is reconstructed after the $k$ th adjective has been presented and $w_{1, k}$ and $w_{0, k}$ is the weights of the $k$ th adjective and the impression before the presentation of this adjective. $w_{1, k} / w_{0, k}$ is assumed to be a decreasing function of $k$. In this article, as a first approximation, let $w_{1, k} / w_{0, k}=a \cdot \exp (-b \cdot k)$. Rewriting the equation (1), we obtain the following model, equation (2), 


$$
R_{k}=\frac{R_{k-1}+a \cdot \exp (-b \cdot k) \cdot S_{k}}{1+a \cdot \exp (-b \cdot k)} .
$$

Equation (2) was applied to Fig. 2.9 in Anderson (1981) with the method of least squares. The experiment, the result of

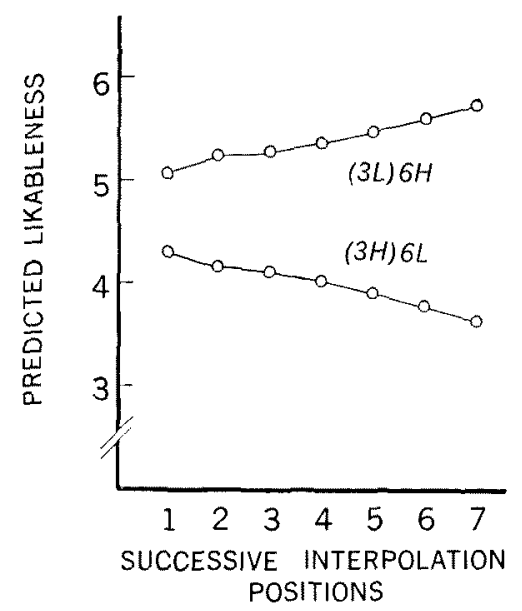

Fig. 1. Predicted primacy effect. Upper (or lower) curve represents predicted responses to sequence of $3 L$ (or $H$ ) and $6 H$ (or $L$ ) adjectives, with the $3 \mathrm{~L}$ 's (or $H^{\prime}$ 's) interpolated as a block at each possible point within the sequence of $6 \mathrm{H}$ 's (or $L$ 's). The parameter values are $a=0.341, b=$ $0.201, S_{H}=7.59, S_{L}=1.73, R_{1 H}=5.57$ and $R_{1 L}=$ 3.88 . which is presented as Fig. 2.9, adopted the final mode of responding, where in (3L) $6 H$ (or $(3 H) 6 L$ ) condition the first to $(k-1)$ th and $(k+3)$ th to 9 th adjectives have high (or low) likableness values and $k$ th to $(k+2)$ th adjectives have low (or high) ones for the successive interpolation position $k$. The subject responded only after the 9 th adjective was presented. The estimated values of parameters are $a=0.341, b=0.201, S_{H}$ (the adjectives of high likableness $)=7.59, S_{L}$ (the adjectives of low likableness) $=1.73$, $R_{1 I I}$ (the impression after the first adjective of high likableness was presented) $=5.57$, and $R_{1 L}$ (the impression after the first adjective of low likableness was presented $)=3.88$. The calculated values of $R_{9}$ 's with these parameters are shown in Fig. 1, which resembles very well to Fig. 2.9 in Anderson (1981) qualitatively.

In the successive mode of responding, the subject responds every time after each additional adjective of a given set was presented. In $H L$ (or $L H$ ) presentation order, the subject was presented adjectives of high (or low) likableness on the first half of the trials and of low (or high) ones on the last half of the trials. It seems that each responding exerts pro-

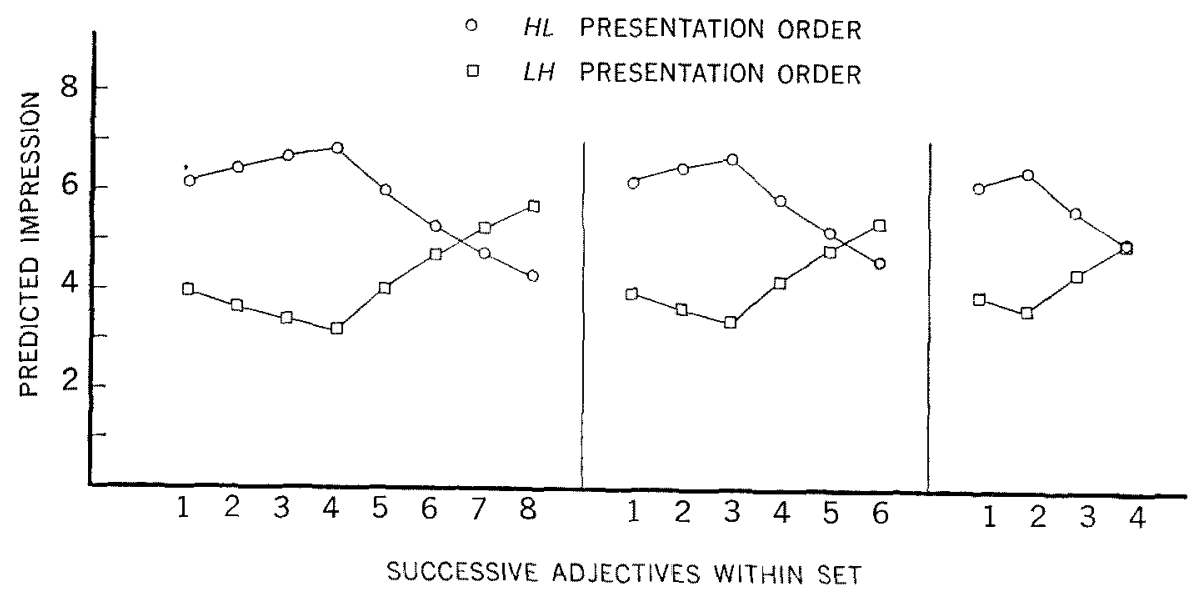

Fig. 2. Predicted serial curves for cumulative response in personality adjective task. Upper (or lower) curves for sequences with favorable (or unfavorable) adjectives first, followed by unfavorable (or favorable) adjectives. The parameter values are $w=0.232, S_{H}=7.60, S_{L}=2.32, R_{1 E}=6.16$ and $R_{1 L}=3.94$. 
found effects on the subject. The subject is forced to verbalize the judgment considering the presented adjective, so it is natural to hypothesize that, in responding, the weight of the presented adjective is constant across every presentation and this verbalized impression becomes the current one which the subject has as his internal one, because even the role playing induces opinion changes (e.g. Janis \& King [1954]). The following equation (3) is proposed as the model for the continuous mode of responding,

$$
R_{k}=\frac{R_{k-1}+w \cdot S_{k}}{1+w}
$$

where $R_{k}$ is the impression which is verbalized after the $k$ th adjective was presented and becomes internal, and $w$ is the weight of the presented adjective. Equation (3) was applied to Fig. 3.5 in Anderson (1981) with the method of least squares. The estimated values of parameters are $w=0.232, S_{H}$ (the adjectives of high likableness) $=7.60, S_{L}$ (the adjectives of low likableness $)=2.32, R_{1 H}$ (the impression after the first adjective of high likableness was presented) $=6.16$, and $R_{1 L}$ (the impression after the first adjective of low likableness was presented) $=3.94$. The calculated values with these estimations by equation (3) are plotted in Fig. 2, which resembles to Fig. 3.5 in Anderson (1981).

\section{Discussion}

Roughly speaking, both Figs. 1 and 2 produced successfully the patterns shown in Figs. 2.9 and 3.5 in Anderson (1981), i.e. the primacy and recency effects. Remembering that the equations (2) and (3) are proposed as first approximations, closer comparison of these figures teaches us several ways to improve the proposed models.

As for Fig. 1, the calculated values of primacy effects for both conditions ( $3 \mathrm{~L}) 6 \mathrm{H}$ and $(3 H) 6 L$ are equal, although in Fig. 2.9 of Anderson (1981) primacy effects differ between two conditions. This difference suggests the possibility that the weights of the adjectives of high and low likableness are different.

As for Fig. 2, the recency effect does not appear for 4-successive-adjective condition. Several points should be considered. The direction of change from the first to second adjectives in this condition with $L H$ presentation order is positive, owing to some disturbance. Another point concerns simplifications in this model. The proposed model neglects contextual effects or biases which might be caused by response and perceptual systems. The subject might respond not only on the base of information integration process, but also under the pressure caused by the experimental procedure by which the subject was presented the same type of adjectives successively.

In general cases, we should also consider other factors than those discussed above, which may have effects on integration and response processes. Future versions of step-by-step types of models will include these features.

\section{References}

Anderson, N.H. 1959 Test of a model for opinion change. Journal of Abnormal and Social Psychology, 59, 371-381.

Anderson, N.H. 1965 Primacy effects in personality impression formation using a generalized order effect paradigm. Journal of Personality and Social Psychology, 2, 1-9.

Anderson, N. H. 1981 Foundations of information integration theory. New York: Academic Press, Inc.

Janis, I. L., \& King, B. T. 1954 The influence of role playing on opinion change. Journal of Abnormal and Social Psychology, 49, 211-218.

(Received April 26, 1984; accepted Sept. 26, 1984) 